'Sección Neurología Hospital Naval Almirante Nef, Viña del Mar, Chile. ${ }^{2}$ Cátedra de Neurología. Escuela de Medicina. Universidad de Valparaíso. Hospital Carlos Van Buren. Valparaíso, Chile Servicio de Medicina. Hospital Almirante Nef Viña del Mar, Chile.

${ }^{4}$ Geroscience Center for Brain Health and Metabolism. Universidad de Chile. Santiago. Chile.

${ }^{5}$ Departamento de Fisiopatología, ICBM y Departamento de Ciencias Neurológicas Oriente, Facultad de Medicina, Universidad de Chile, Santiago, Chile.

${ }^{6}$ Unidad de Neurología Cognitiva y Demencias, Servicio de Neurología, Hospital del Salvador, Santiago, Chile.

${ }^{7}$ Centro de Investigación Avanzada en Educación, Universidad de Chile, Santiago, Chile.

${ }^{8}$ Servicio de Neurología, Clínica Alemana Santiago, Chile. ${ }^{9}$ Corporación Profesional de Alzheimer y Otras Demencias COPRAD Chile.

${ }^{10} \mathrm{Centro}$ de Investigaciones BiomédicasUniversidad de Valparaíso. Valparaíso, Chile.

Trabajo finaciado por proyectos: CONICYT /FONDECYT/ 1140423 (JI-AS);

CONICYT / FONDAP /15150012 (AS); \& Basal Funds for Centers of Excellence, Project FB 0003 from the Associative Research Program of CONICYT (AS)

Proyecto DIUV 17/2011 Universidad de Valparaíso.

Recibido el 24 de marzo de 2016, aceptado el 10 de enero de 2017.

Correspondencia a: Dr. Juan Idiáquez Hortaneda 1653. Valparaíso, Chile. Teléfono: 322507695 idiaquez@123.c

\section{Cuestionario de actividades de la vida diaria (T-ADLQ): utilidad en pacientes con accidente cerebrovascular menor}

\author{
JUAN IDIÁQUEZ ${ }^{1,2}$, FRANCISCO TORRES $^{2}$, EVA MADRID $^{2,10}$, \\ JORGE VEGA ${ }^{2,3}$, ANDREA SLACHEVSKY ${ }^{4-9}$
}

\section{Usefulness of the activities of daily living questionnaire (T-ADLQ) in patients with minor stroke}

Background: The inability to carry out activities of daily living $(A D L)$ is prevalent in elderly people and it is associated with hypertension and stroke. Aim: To evaluate ADLs using the T-ADLQ in hypertensive patients with minor stroke. Subjects and Methods: T-ADLQ, Cognitive tests (Minimental and Addenbrooke), and Hamilton depression test were applied to 100 hypertensive ambulatory patients (55 without symptomatic stroke and 45 with ischemic stroke, Rankin $\leq 2)$. Results: In stroke patients the ability to perform $A D L$ was significantly reduced compared with hypertensive patients without stroke. Cognitive dysfunction and depressive symptoms were associated with a lower ADL performance. Conclusions: The T-ADLQ is useful to evaluate ADL in hypertensive ambulatory patients with ischemic stroke.

(Rev Med Chile 2017; 145: 188-193)

Key words: Activities of Daily Living; Hypertension; Stroke; Surveys and Questionnaries.
$\mathrm{E}$ 1 número de pacientes sobrevivientes a un accidente cerebrovascular (ACV) y la carga global del ACV son altos y ha ido en aumento $^{1-3}$. La discapacidad y morbilidad asociadas al AVC disminuyen la calidad de vida ${ }^{4}$. A proximadamente $30 \%$ presenta deterioro cognitivo vascular y $50 \%$ de los pacientes que sobreviven a un ACV tiene discapacidad importante 6 meses después del episodio agudo ${ }^{5}$. La hipertensión arterial (HTA) es una de las principales causas de ACV y que, en forma independiente o en conjunto con la ocurrencia de ACV sintomáticos, puede asociarse a déficit cognitivo, con deterioro de las actividades de la vida diaria $(\mathrm{AVD})^{6}$.
El compromiso de las AVD en pacientes hipertensos con ACV determina un deterioro en la independencia y funcionalidad. Para evaluar las AVD en pacientes con ACV se ha utilizado el índice de Barthel, que enfatiza la medición de los rendimientos reales como factor de valoración de las $\mathrm{AVD}^{7,8}$. Este índice muestra buenas propiedades psicométricas en términos de validez y confiabilidad. Sin embargo, este instrumento evalúa principalmente las actividades básicas de la vida, omitiendo la evaluación de las actividades instrumentales ${ }^{9}$. El cuestionario T-ADLQ, validado en español, incluye ítems para AVD básicas e instrumentales, evaluando el desem- 
peño en seis áreas (autocuidado, actividades en el hogar, trabajo, recreación, manejo de dinero, viajes y comunicación y tecnologías de la información $)^{10,11}$. El T-ADLQ se construyó a partir del ADLQ añadiendo un dominio de tecnología ${ }^{11}$. El T-ADLQ es una escala válida y confiable para la evaluación funcional de pacientes con deterioro cognitivo asociado a demencias ${ }^{12}$. El T-ADLQ, al valorar en forma detallada distintas AVD, puede ser útil en pacientes con limitaciones cognitivas o motoras leves. Este cuestionario no ha sido utilizado en pacientes con ACV menor, por lo tanto, el objetivo de este estudio fue evaluar los distintos déficits funcionales que pueden afectar AVD en pacientes hipertensos sin y con presencia de un $\mathrm{ACV}$ isquémico menor sintomático.

\section{Pacientes y Método}

Se seleccionó para este estudio a 45 pacientes adultos (mayores de 18 años) hipertensos que hubieran experimentado un ACV sintomático durante los 3 a 12 meses previos. Grupo control fueron pacientes hipertensos que no habían sufrido un AVC. Se incluyó pacientes con cuadro clínico, hallazgos anormales en el examen neurológico y un estudio de imágenes (tomografía axial computarizada de cerebro o resonancia magnética) compatibles con infarto cerebral. Los pacientes eran de los hospitales Carlos Van Buren (Valparaíso) y Naval Almirante Nef (Viña del Mar). La investigación se desarrolló entre octubre de 2011 y febrero de 2012.

\section{Criterios de exclusión}

1) Pacientes con enfermedades neurológicas previas al AVC, enfermedades psiquiátricas como demencia, esquizofrenia u otros trastornos psicóticos, enfermedades sistémicas graves como neoplasias terminales, insuficiencia cardiaca, hepática o renal graves; 2) Pacientes con ACV con una puntuación en escala de Rankin mayor a $2^{13}$.

El estudio fue aprobado por los comités de ética de ambos hospitales.

Se registró: género, edad, nivel de escolaridad, presencia de HTA, diabetes mellitus, dislipidemia e índice de masa corporal (IMC). Las escalas de evaluación y pruebas cognitivas efectuadas fueron:

1. Pruebas de Evaluación Cognitiva: El MMSE (prueba Minimental de Folstein) es una valora- ción general de las funciones cognitivas. A menor puntaje, mayor deterioro cognitivo ${ }^{14}$. El ACE (Addenbrooke's cognitive examination) es una prueba que evalúa 5 dominios cognitivos, un puntaje más bajo indica mayor deterioro cognitivo ${ }^{15}$.

2. Escala de depresión: Escala de Hamilton ${ }^{16}$ con puntuación de síntomas: 0 a 7 "normal", 8 a 13 "leve", 14 a 18 "moderado", 19 a 22 "severo" y 23 o más "muy severo".

3. Escala de Funcionalidad. (AVD): T-ADLQ; Esta prueba evalúa 7 áreas: autocuidado (6 ítems), cuidado y manejo del hogar (6 ítems), trabajo y recreación (4 ítems), compras y dinero (3 ítems), viajes (3), comunicación (5 ítems) y tecnología (5). Cada ítem tiene un puntaje, donde 0 es no tener problemas para la actividad hasta 3 que indica que no puede realizar la actividad. Presenta flexibilidad al no puntuar aspectos que no forman parte de las actividades del paciente. Así, existe además un ítem 9, que pregunta si el paciente nunca realizó la actividad o si no realiza la actividad desde antes de sufrir el ACV y también se considera si el paciente no tiene información precisa sobre la actividad que se le interroga. El déficit funcional se calcula para cada área y para el cuestionario global, de acuerdo a la fórmula: suma de todos los puntajes ( $\sin$ el ítem 9) / $3 \mathrm{x}$ número total de ítems. Un puntaje más alto indica un mayor deterioro de las AVD ${ }^{12}$. Esta prueba se encuentra validada en español ${ }^{11,12}$.

4. Escalas de Evaluación de ACV: La escala de Rankin modificada para discapacidad global del $\mathrm{ACV}^{13}$, la escala del National Institutes of Health Stroke Scale (NIHSS) ${ }^{3}$ para medir severidad del $\mathrm{ACV}$ y clasificación del tipo de ACV isquémico de Oxfordshire, según acrónimos anglosajones (LACI $=$ infarto lacunar, $\mathrm{PACI}=$ infarto parcial de la circulación anterior, POCI = infarto de la circulación posterior, TACI = infarto total de la circulación anterior) ${ }^{17}$.

El diagnóstico de ACV y de HTA se hizo de acuerdo a la guía clínica del MINSAL ${ }^{18}$.

Se realizó análisis descriptivo, describiendo variables cualitativas con frecuencias y porcentajes y variables cuantitativas con medias y desviación estándar. Se empleó estadística analítica para determinar la asociación entre el antecedente de 
ACV isquémico sintomático y el compromiso de las AVD mediante la prueba exacta de Fisher. Para comparar los promedios de T-ADLQ entre ambos grupos se utilizó el test T de Student. Correlación entre las variables con la prueba de Pearson. Software STATA 12.0 SE (Statacorp USA).Se utilizó un nivel de significancia alfa de 0,05 .

\section{Resultados}

La Tabla 1 muestra los datos demográficos y clínicos de los pacientes, separados en 2 grupos de acuerdo a la presencia o ausencia de ACV isquémico. En relación a la edad, escolaridad e IMC no se encontraron diferencias entre ambos grupos. El sexo femenino fue más frecuente en los pacientes sin antecedentes de ACV. En los pacientes con ACV, el NIHSS promedio fue 3,4 puntos y $51 \%$ tenía un infarto lacunar y no se incluyeron pacientes con infartos totales (TACI). La escala de Rankin mostró, en el grupo con ACV, los siguientes puntajes: 0 en 4,4\%, 1 en $57,8 \%$ y 2 en $47,8 \%$ de los pacientes.

Se encontró compromiso funcional significativo de las AVD en el grupo con ACV en todos los ítems (Tabla 2).

La severidad del ACV medida por la escala de NIHSS mostró una correlación positiva con el mayor compromiso funcional medido por el T-ADLQ $(\mathrm{r}=0,6, \mathrm{p}<0,001)$ La discapacidad global del ACV medida por la escala de Rankin modificada mostró una correlación positiva con el puntaje del T-ADLQ $(\mathrm{r}=7, \mathrm{p}<0,001)$.

Tabla 1. Características clínicas y demográficas de pacientes con y sin ACV

\begin{tabular}{|c|c|c|c|c|c|}
\hline Variables & & $\begin{array}{l}\text { Sin ACV } \\
(n=55)\end{array}$ & $\begin{array}{l}\text { Con ACV } \\
(n=45)\end{array}$ & $\begin{array}{c}\text { Total } \\
(n=100)\end{array}$ & $\mathbf{p}$ \\
\hline Edad & media (DE) & $65(15)$ & $65,8(11)$ & $65,5(14)$ & $0,811^{*}$ \\
\hline $\begin{array}{l}\text { Sexo } \\
\text { Femenino }\end{array}$ & $\%$ & 65,5 & 37,8 & 53 & $0,009 * *$ \\
\hline $\begin{array}{l}\text { Escolaridad (años) } \\
0-4 \\
5-8 \\
9-12 \\
>12\end{array}$ & $\begin{array}{l}\text { n (\%) } \\
\text { n (\%) } \\
\text { n (\%) } \\
\text { n (\%) }\end{array}$ & $\begin{array}{rr}5 & (9,1) \\
12 & (21,8) \\
21 & (38,2) \\
17 & (30,9)\end{array}$ & $\begin{array}{r}8(17,8) \\
6(13,3) \\
24(53,3) \\
7(15,6)\end{array}$ & $\begin{array}{ll}13 & (13) \\
18 & (18) \\
45 & (45) \\
24 & (24)\end{array}$ & $0,1^{* *}$ \\
\hline IMC & media (DE) & $27,2(3,1)$ & $26,7(3,2)$ & $26,9(3,1)$ & 0,44 \\
\hline Diabetes mellitus & $(\%)$ & 14,6 & 31 & 22 & 0,055 \\
\hline $\begin{array}{l}\text { Hipertensión arteri } \\
\text { Grado I } \\
\text { Grado II y III }\end{array}$ & $\begin{array}{l}\% \\
\%\end{array}$ & $\begin{array}{l}85,5 \\
14,6\end{array}$ & $\begin{array}{l}84,4 \\
15,6\end{array}$ & $\begin{array}{l}85 \\
15\end{array}$ & 1 \\
\hline Dislipidemia & $(\%)$ & 23,6 & 20 & 22 & 0,809 \\
\hline NIHSS & media (DE) & & $3,42( \pm 2$ & & \\
\hline $\begin{array}{l}\text { Rankin m } \\
0 \\
1 \\
2\end{array}$ & $\begin{array}{l}\% \\
\% \\
n(\%)\end{array}$ & $\begin{array}{r}97 \\
3 \\
0\end{array}$ & $\begin{array}{r}4,4 \\
57,8 \\
37,8\end{array}$ & $\begin{array}{l}52 \\
28 \\
20\end{array}$ & \\
\hline $\begin{array}{l}\text { Tipo de infarto }(0 x \\
\text { LACI } \\
\text { PACI } \\
\text { POCI } \\
\text { TACI }\end{array}$ & $\begin{array}{l}\text { rdshire) } \\
\% \\
\% \\
\% \\
\%\end{array}$ & & $\begin{array}{l}51 \\
31,1 \\
17,8 \\
0\end{array}$ & & \\
\hline
\end{tabular}

*Prueba T de Student. **Prueba exacta de Fisher. IMC = Índice de masa corporal. NIHSS = National Institute of Health Stroke Scale. Rankin $m=$ Escala de Rankin modificada (discapacidad global). Clasificación de subtipo de infarto = LACl: lacunar, $\mathrm{PACl}$ : parcial circulación anterior, $\mathrm{POCl}$ : parcial circulación posterior, $\mathrm{TACl}$ : total circulación anterior. 
Tabla 2. Actividades de la vida diaria (T-ADLQ) , pruebas cognitivas y síntomas de depresión en pacientes hipertensos sin y con ACV isquémico

\begin{tabular}{|c|c|c|c|c|}
\hline Variable & & Sin ACV & Con ACV & $\mathbf{p}$ \\
\hline ADLQ-T & media (DE) & $6,2 \quad(7,5)$ & $23,7 \quad(22,8)$ & $<0,0001^{*}$ \\
\hline Autocuidado & & $0,01 \quad(0)$ & $(0,2)$ & $<0,0001^{*}$ \\
\hline Hogar & & $0,07 \quad(0,1)$ & $13,2 \quad(0,4)$ & $<0,0001 *$ \\
\hline Trabajo y recreo & & $0,1 \quad(0,2)$ & 19,7 & $<0,0001 *$ \\
\hline Compra y dinero & & $0,04 \quad(0,2)$ & $(0,3)$ & $<0,0001^{*}$ \\
\hline Viajes & & $0,1 \quad(0,1)$ & 16,1 & $<0,0001^{*}$ \\
\hline Comunicación & & $0,02 \quad(0)$ & $(0,2)$ & $<0,0001^{*}$ \\
\hline Tecnología & & $(0,2)$ & $(0,2)$ & $<0,0001^{*}$ \\
\hline MMSE & media (DE) & $27,9 \quad(2,5)$ & $25,8 \quad(4,1)$ & $0,0025^{*}$ \\
\hline ACE & media (DE) & $84,4 \quad(10)$ & $76,5 \quad(13,3)$ & $0,0012^{*}$ \\
\hline Hamilton & media (DE) & $(3,8)$ & $(5,4)$ & $0,0005^{*}$ \\
\hline
\end{tabular}

*Prueba T de Student y prueba de Bonferroni que da un valor de $p \leq 0,0045$ estadísticamente significativo. T-ADLQ = Cuestionario de actividades de la vida diaria. MMSE = Prueba Minimental de Folstein. ACE = Prueba de Addenbrooke. Hamilton $=$ escala de depresión de Hamilton.

En la evaluación con las pruebas de función cognitiva: En el grupo de pacientes con ACV los cuestionarios MMSE y ACE mostraron un rendimiento cognitivo inferior al grupo de pacientes hipertensos sin ACV. Ninguno de los pacientes con antecedente de ACV tenía antecedentes clínicos de demencia. En el grupo con ACV se encontró una correlación entre el mayor compromiso funcional de las AVD y el menor rendimiento cognitivo en las pruebas de MMSE $(r=-0,7, p<0,001)$ y ACE $(\mathrm{r}=-0,7<0,001))$

Un análisis de regresión múltiple mostró que el puntaje total del T-ADLQ se correlacionó con el puntaje de los rendimientos cognitivos (MMSE y ACE), en forma independiente, controlando edad y escolaridad (años de estudio): para MMSE: $\mathrm{R} 2=0,5$, para ACE: $\mathrm{R} 2=0,5)$.

El puntaje de la escala de Hamilton para depresión mostró más síntomas de depresión en el grupo con AVC sintomático (Tabla 2). Se encontró un puntaje de síntomas de 8 o más en $35,5 \%$ de los pacientes con ACV sintomático y en 12,7\% de los pacientes hipertensos sin ACV $(\mathrm{p}=0,009)$.

$\mathrm{Al}$ considerar la relación entre el compromiso de las AVD y la escala de depresión de Hamilton hubo $4,81( \pm 4,20)$ puntos de promedio en los pacientes sin compromiso de las AVD versus 10,85 $( \pm 5,69)$ puntos de promedio en los examinados con compromiso de las AVD ( $\mathrm{p}<0,0001)$.

\section{Discusión}

El presente estudio muestra que el cuestionario sobre actividades de la vida diaria T-ADLQ validado en español, en pacientes con deterioro cognitivo $^{10-12}$, permite evaluar el compromiso funcional de las AVD en pacientes hipertensos ambulatorios con antecedente de ACV isquémico. Nuestro grupo de pacientes con ACV tenían un puntaje de severidad del NHISS promedio de 3,4, correspondiendo a un ACV menor ${ }^{19}$. El T-ADLQ es un cuestionario que, además de los ítems de autocuidado, hogar, trabajo y recreación, compras y dinero, viajes y comunicación, agrega un ítem sobre manejo de tecnología ${ }^{12}$. El cuestionario T-ADLQ permitió cuantificar el grado de compromiso, mostrando en el grupo de pacientes con ACV un menoscabo significativo en los ítems de las distintas actividades, en relación al grupo sin ACV El deterioro de las AVD, medido con el T-ADLQ, se asoció con la severidad del ACV, la presencia de síntomas depresivos y de trastorno cognitivo.

En pacientes con ACV se han utilizado distintos cuestionarios para evaluar las AVD, principalmente el índice de Barthel, que mide los rendimientos reales ${ }^{7,8}$. El índice de Barthel presenta una baja sensibilidad para mostrar cambios pequeños en el estado funcional del paciente ${ }^{9}$. También existe un instrumento para evaluar la 
independencia funcional (FIM) del paciente, el cual, además de medir las AVD, evalúa aspectos motores y cognitivos. El FIM es una herramienta utilizada en el seguimiento de pacientes con distintas patologías durante la terapia de rehabilitación ${ }^{20}$. El T-ADLQ evalúa en forma más detallada las AVD y fue útil en nuestro grupo de pacientes ambulatorios con ACV, quienes presentaban un déficit neurológico menor. Recientemente se han agregado mediciones de la autopercepción y de las capacidades funcionales ${ }^{21}$.

Nuestro estudio muestra que el compromiso funcional de las AVD en pacientes hipertensos con ACV sintomático menor se relaciona con un mayor deterioro del rendimiento cognitivo. Aunque ningún paciente cumplía los criterios clínicos demencia ${ }^{22}$, el grupo con ACV tenía un rendimiento menor en las pruebas cognitivas (MMSE y ACE). El grupo de pacientes con ACV no presentaba diferencias significativas de edad ni de nivel de escolaridad, con el grupo de pacientes hipertensos sin ACV, por lo que estos factores no influyeron en la diferencia de los rendimientos cognitivos.

En este estudio, el grupo de pacientes con ACV presentó un puntaje de síntomas de depresión más alto que en el grupo sin ACV. Se encontró una asociación entre los síntomas de depresión y mayor compromiso de las AVD. Se ha comunicado que en el periodo posterior al ACV existe una asociación entre la presencia de depresión y el mayor compromiso funcional de las $\mathrm{AVD}^{23}$.

Los resultados de nuestro estudio son concordantes con estudios previos que muestran que el deterioro de la calidad de vida en pacientes ACV depende de distintos factores, como el grado de dependencia física, los trastornos neuropsiquiátricos (depresión y otros), el deterioro de la comunicación social y el grado de dependencia en las $\mathrm{AVD}^{23,24}$.

Una limitación del estudio es que el cuestionario T-ADLQ mide el deterioro funcional de las AVD basado en la información que se obtiene de un informante, de modo que este puede sobreestimar o subvalorar el deterioro funcional del paciente, considerando que los pacientes con ACV pueden presentar un compromiso cognitivo selectivo o un déficit motor o sensitivo localizado.

En conclusión, este estudio muestra que la aplicación del cuestionario T-ADLQ permite valorar el deterioro funcional de pacientes con
ACV menor, y que la asociación de mayor compromiso de actividades de la vida diaria con la presencia de deterioro cognitivo y síntomas depresivos es un hallazgo que debe ser considerado en el manejo conjunto de la evaluación, terapia y rehabilitación.

\section{Referencias}

1. Feigin VL, Forouzanfar MH, Krishnamurthi R, Mensah GA, Connor M, Bennett DA, et al. Global Burden of Diseases, Injuries, and Risk Factors Study 2010 (GBD 2010) and the GBD Stroke Experts Group. Global and regional burden of stroke during 1990-2010: findings from the Global Burden of Disease Study 2010. Lancet 2014; 383 (9913): 245-54.

2. Lavados PM, Sacks C, Prina L, Escobar A, Tossi C, Araya $\mathrm{F}$ et al. Incidence, 30-day case-fatality rate, and prognosis of stroke in Iquique, Chile: a 2-year community-based prospective study (PISCIS project). Lancet 2005; 365 (9478): 2206-15.

3. Guía clínica. Accidente cerebrovascular isquémico en personas mayores de 15 años o más. Serie Guias Clínicas. MINSAL,Santiago. Chile; 2013. p. 11-12. www. bibliotecaminsal.cl

4. Muus I, Petzold M, Ringsberg KC. Health-related quality of life among Danish patients 3 and 12 months after TIA or mild stroke. Scand J Caring Sci 2010; 24 (2): 211-8.

5. Mayo NE, Wood-Dauphinee S, Cote R, Durcam, L Carleton J. Activity, Participation, and quality of life 6 months post stroke. Arch Phys Med Rehabil 2002; 83 (8): 1035-42.

6. Qiu C, Winblad B, Fratiglioni L. The age-dependent relation of blood pressure to cognitive function and dementia. Lancet Neurol 2005; 4 (8): 487-99.

7. Quinn TJ, Langhorne P, Stott DJ. Barthel index for stroke trials: development, properties, and application. Stroke 2011; 42 (4): 1146-51.

8. Hsieh CL, Hoffmann T, Gustafsson L, Lee YC. The diverse constructs use of activities of daily living measures in stroke randomized controlled trials in the years 2005-2009. J Rehabil Med 2012; 44: 720-6.

9. Hsueh I, Wang W, Sheu C, Hsieh C. Rasch analysis of combining two indices to assess comprehensive ADL function in stroke patients. Stroke 2004; 35 (3): 721-6.

10. Johnson N, Barion A, Rademaker A, Rehkemper G, Weintraub $S$. The activities of daily living questionnaire: a validation study in patients with dementia. Alzheimer Dis Assoc Disord 2004; 18 (4): 223-30. 
11. Gleichgerrecht E, Camino J, Roca M, TorralvaT, Manes F. Assessment of functional impairment in dementia with the Spanish version of the Activities of Daily Living Questionnaire. Dement Geriatr Cogn Disord 2009; 28 (4): 380-8.

12. Muñoz-Neira C, Lopez OL, Riveros R, Nuñez-Huasaf J, Flores P, Slachevsky A. The 'Technology Activities of Daily Living Questionnaire': a version with a technology-related subscale. Dement Geriatr Cogn Disord 2012; 33 (6): 361-71.

13. Kasner S. Clinical interpretation and use of stroke scales. Lancet Neurol 2006; 5 (7): 603-12.

14. Folstein MF, Folstein SE, McHugh PR. Mini-mental state examination: a practical method for grading the cognitive state of patients for the clinician. J Psychiatr Res 1975; 12 (3): 189-98.

15. Muñoz-Neira C, Henríquez F, Ihnen J, Sanchez M, Flores P, Slachevsky A. Propiedades psicométricas y utilidad diagnóstica del Addenbrooke’s Cognitive Examination-Revised (ACE-R) en una muestra de ancianos chilenos Rev Med Chile 2012; 140 (3): 1006-13.

16. Hamilton M.Development of a rating scale for primary depressive illness. Brit J Soc Clin Psychol 1967; 6 (4): 278-96.

17. Díez-Tejedor E, Del Brutto O, Álvarez-Sabín J, Muñoz M, Abiusi G. Clasificación de las enfermedades cerebrovasculares. Sociedad Iberoamericana de Enfermedades
Cerebrovasculares Rev Neurol 2001; 33 (5): 455-64.

18. Guía clínica. Hipertensión arterial primaria o esencial en personas de 15 o más años. MINSAL. Santiago. Chile;2010.p 11-13. www.minsal.gob.cl

19. Fischer U, Baumgartner A, Arnold M, Nedeltchev K, Gralla J, De Marchis GM et al. What is a minor stroke? Stroke 2010; 41 (4): 661-6.

20. Chumney D, Nollinger K, Shesko K, Sop K, Spencer $\mathrm{M}$, Newton RA. Ability of Functional Independence Measure to accurately predict functional outcome of stroke-specific population: systematic review. J Rehabil Res Dev 2010; 47 (1): 17-29.

21. Lee Y-C, Chen S-S, Koh C-L, Hsueh I-P, Yao K-P,Hsieh CL. Development of Two Barthel Index-Based Supplementary Scales for Patients with Stroke. PLoS ONE 2014; 9 (10): e110494.

22. American Psychiatric Association: Diagnostic and Statistical Manual of Mental Disorders (DSM-IV-TR). Barcelona, España: Editorial Masson.; 2000. p 139-43.

23. Haghgoo HA, Pazuki ES, Hosseini AS, Rassafiani M. Depression, activities of daily living and quality of life in patients with stroke J Neurol Sci 2013; 328 (1-2): $87-91$.

24. Kim K, Kim YM, Kim EK. Correlation between the Activities of Daily Living of Stroke Patients in a Community Setting and Their Quality of Life. J Phys Ther Sci 2014; 26 (3): 417-9. 